\title{
Le lexique du chat sur Internet : étude comparative français-espagnol-polonais
}

\author{
Lorenz, Paulina \& Michot, Nicolas \\ Université Paris V Sorbonne \& LDI-Cergy \\ lorenzpaulina@yahoo.fr \\ nicolasmichot1@yahoo.fr
}

\section{Introduction}

Le chat sur Internet est un moyen moderne de communication électronique ayant engendré de nouvelles pratiques d'écriture chez les usagers. Cet outil de communication emblématique est généralement connu pour les procédés graphiques de réduction, le caractère phonétique de l'écriture, ou encore les procédés d'expressivité. Ceci étant, si ces procédés graphiques sont les caractéristiques linguistiques les plus décrites $^{1}$ et les plus saillantes des discours chatiens, certains phénomènes tels que l'usage de lexiques particuliers et diversifiés ne méritent pas moins d'attention. Nous décrirons donc les usages des lexiques utilisés dans les chats par les différents types d'intervenants afin d'en déterminer les spécificités. Ainsi, nous pouvons nous demander quelle est la diversité des lexiques et s'ils sont spécifiques à ce moyen de communication. Nous nous demanderons de plus quels sont les liens entre l'oral et l'écrit.

La réflexion que nous avons choisi de conduire dépasse le cadre de la description propre à une seule langue puisque nous nous intéressons comparativement au français, à l'espagnol et au polonais. Il est en effet intéressant de se demander si le langage du chat est un phénomène globalisant présentant des universaux linguistiques. Ainsi, la problématique de l'étude des lexiques englobera les questions suivantes : Les langues française, espagnole et polonaise fonctionnent-elles de la même manière dans les chats ? Quels sont les phénomènes lexicaux récurrents et divergents d'une langue à l'autre ? Est-il possible de parler d'une identité de cybercommunauté chatienne sur le plan lexical ?

Nous commencerons notre étude par la présentation de notre corpus de chat avant de décrire le lexique à travers deux grandes catégories que nous proposons : le fonds du lexique commun et le fonds du lexique chatien. Enfin, par extension au lexique nous décrirons les smileys utilisés dans le chat dans la mesure où ils constituent une spécificité chatienne, et où ils représentent des séquences graphiques jouant un rôle sur le plan du sens.

\section{Corpus}

L'étude des lexiques du chat sera fondée sur un corpus relevé sur trois sites : français, www.yahoo.fr, espagnol, www.yahoo.es et polonais, www.interia.pl. Ces trois sites sont des chats publics, c'est-à-dire ouverts à tous, et présentent deux possibilités de discussion : la discussion publique via le canal ouvert à tous les chateurs, et la discussion privée se déroulant de façon confidentielle entre les chateurs du canal. Notre corpus présente uniquement des conversations publiques car il nous est impossible d'accéder aux conversations privées. Les sites de chat ont été choisis en fonction de certains critères. D'abord, chacun des sites des trois langues devait être représentatifs des pratiques chatiennes propre à chacune d'elle. Ensuite, les sites devaient être comparables au niveau des communicants, et de ce fait, le choix le plus concluant était celui de l'ouverture à un public diversifié. Ainsi, on a retenu les chats yahoo Espagne et France qui fonctionnent de la même façon et qui présentent des similitudes sur le plan des fréquentations, ainsi qu'interia en polonais qui est un chat à la mode et fréquenté par un public ouvert. Au niveau de la taille, le corpus est équilibré entre le français (16 extraits de discussions), l'espagnol (10 extraits de discussions), et le polonais (11 extraits de discussions). Il est à préciser que la taille des discussions est variable même s'il s'agit globalement d'extraits longs, et la diversité est équivalente d'un chat à l'autre même lorsque moins de discussions ont été retenues dans la mesure où l'une des particularités d'un chat 
public est le roulement régulier des participants. Sur le plan des répliques des chateurs, on peut dénombrer un total d'un millier de répliques environ pour les trois langues.

En ce qui concerne nos enregistrements, aucune modification n'a été opérée de notre part. Autrement dit, on a copié de façon "brute» les discussions des chateurs par souci d'objectivité. Lors de citations d'exemples au sein de nos analyses, on conservera donc le texte tel qu'il se présente dans les conversations, c'est-à-dire avec ses procédés et tous ses phénomènes graphiques, lexicaux et syntaxiques sans modification, ni censure de notre part. De la même façon, les tours de parole seront conservés lorsqu'un extrait de discussion sera cité. Précisons que concernant l'analyse du lexique, dans plusieurs cas, les mots seront cités en dehors de leur contexte d'apparition.

\section{Fonds du lexique commun}

Nous désignons par fonds du lexique commun l'ensemble du lexique utilisé dans le chat qui se compose de divers registres. Ce lexique qui apparaît habituellement dans les usages quotidiens est d'une manière naturelle employé aussi dans le chat.

Le chat présente une situation de communication particulière mettant les chateurs provenant potentiellement de divers milieux sociaux sur un pied d'égalité. Ceci étant, l'influence culturelle de chacun et la dynamique de groupe instaurée dans chacune des discussions contribueront indubitablement à orienter le discours, et en particulier, l'usage de tel ou tel registre langagier.

Afin de caractériser les argots du français, Jean-Pierre Goudaillier (2011, à paraître) avance différents critères d'analyse à prendre en considération. Il énumère ainsi :

1) la (les) population(s) concernée(s);

2) la (les) situation(s) relevée(s);

3) la (les) thématique(s) abordée(s) ;

4) la (les) fonction(s) utilisée(s) ;

5) le (les) procédé(s) mis en œuvre (Goudaillier, 2011, à paraitre).

Dans le cas du chat, si le lieu et la situation de communication sont déterminés, la population est difficilement mesurable dans sa diversité. Il peut y figurer des chateurs jeunes, moins jeunes, provenant de tous milieux sociaux. Cependant, la forme, la fonction, et les procédés sont observables, ce qui nous conduit à différencier les lexiques marqués tels que les lexiques populaires et le lexique argotique contemporain des jeunes.

\subsection{Usages populaires}

Les registres argotique, populaire, courant et familier présentent très souvent des limites imprécises fondées sur des jugements de valeur. Ainsi, dans les usages populaires, on se propose de distinguer un ensemble de termes difficilement classifiables représentant les mots familiers, ou les mots non standard provenant de l'argot commun, pour reprendre les termes de Goudaillier (2011, à paraître), des vulgarismes et des interjections.

\subsubsection{Mots non standards}

Les usages de mots non-conventionnels relevant des registres argotiques non marqués «jeune », populaire et vulgaire sont utilisés par tous types de chateurs jeunes et moins jeunes, et provenant de divers milieux sociaux. Dans le corpus français, on en énumère une multitude et l'on se propose de les classer comme relevant des usages populaires. Il est à préciser que ces mots font tous partie de l'oral spontané du quotidien quel que soit le registre. Autrement dit, il s'agit de termes usuels pour les chateurs, quel que soit l'origine sociale. On peut citer : mec (homme); nana (femme); baffe (gifle); pote (camarade, ami, poteau) ; chialer (pleurer, gémir, se plaindre) ; brancher, Ça me branche (ça m'intéresse) ; bidon (faux) ; rechoper (prendre) Avant que je rechope le net ; crever (mourir) ; bourrer le mou (bourrer le crâne, men- 
tir pour tromper) ; pognon (argent) ; en avoir marre (en avoir assez, être excédé) ; naze (nul) ; je me tire (je m'en vais); souler (énerver); rosser (frapper); beulete (belette, jolie fille). Voici des exemples d'occurrences de certains de ces mots ou expressions dans les discours chatiens français :

(1) Babeloula: salut ca te branche un délire en cam ?

(2) Mlle MaHBouLa ChanTeuZ: bon aller je me tire Mlle_MaHBouLa_ChanTeuZ: salam alikoum BENYA_ bye

(3) LANCIEN_PATRON_2_SKY: fait pas le malin toi LANCIEN_PATRON_2_SKY: ou jte ross Bebey-top-chrono: Md'rrrr

De manière analogue, le chat espagnol présente des termes populaires tels que tío, tía ('oncle, tante'. Désigne n'importe quelle personne, signifie aussi 'ami, camarade, mec') ; currar ('bosser'); frío que pela ('il fait très froid'); molar ('plaire') ; tarado ('fou') ; tronco ('ami, camarade') ; macho, machote ('homme') ; pirarse, largarse ('se casser, ficher le camp') ; ostras (interjection qui exprime 'la surprise' ou bien 'la colère'. Ostras se substitue au terme hostia) ; borrico (au sens propre 'l'âne', veut dire 'stupide') ; canuto ('pétard') ; chorradas ('bêtises') ; morro (au sens propre 'museau', fig. 'lèvres') ; dar beso en el morro ('donner un bisou aux lèvres') ; chungo ('nul') ; peña ('bande d'amis') ; partirse de risa ('se tordre de rire'). Voici quelques exemples de mots en discours :

(4) vampikim: maburro tanto ke me voy a poner a currar xdddd ('je m'ennuie tant que je vais me mettre à bosser')

(5) teclas63: agur me piro ('teclas63 au revoir je me casse')

fly_69: teclas63 besos, buen finde ('fly_69: teclas63 bisous, bon weekend')

Enfin, le chat polonais présente aussi des termes propres à la langue orale comme : ziom ('pote, ami') Ce mot au sens propre désigne quelqu'un qui vient du même pays. Ziom est très répandu dans la culture de hip-hop et indique quelqu'un qui provient du même quartier ou bien quelqu'un qui est membre du même groupe de hip-hop; dekiel ('stupide, idiot') mais signifie aussi 'la tête' dans l'expression : masz coś z deklem ? ('t'es fou?') ; kojo ('lit') dans l'expression idę na kojo ('je vais au pieux') ; zawijać, spadać ('se casser') ; palić gumy (signifie 'faire crisser les pneus en démarrant la voiture'. L'expression : pal gumy! 'brûle les pneus', signifie : 'casse-toi') ; lecieć w gumy ('se moquer de quelqu'un, encourir la défaveur de quelqu'un') ; wrzucać ('insulter quelqu'un'). (L'expression wrzucać na starszych veut dire 'insulter les parents') ; laska, lasencja ('une fille sexy'; au sens propre laska est une 'canne') ; cwel ('un homme stupide', ce mot provient du jargon des prisonniers et indique la victime des viols homosexuels) ; lipa ('friperie', au sens propre 'un tilleul'). Il est intéressant de remarquer à ce propos que dans le chat polonais avoir recours aux noms des arbres a une connotation négative. Ainsi, lipa est une 'friperie', czereśniak vient du cerisier et est 'un homme enrichi', kasztan, 'châtaigner' signifie 'merde'. Certes, on dit chłop jak dąb ('un homme comme un chêne', mais on ajoute ironiquement jajka jak żołędzie ('les testicules comme des glands') ; ciacho (au sens propre signifie 'un gâteau', 'c'est un homme qui est beau' ; wyć ('charter mauvais'). Voici quelques exemples de ces mots tirés de leur contexte d'apparition :

(6) oldschoolowy: jepppac kurfy z lksu hej hej ('baiser les putes de ŁKS oé oé') DBW_ON_85: oldschoolowy----- masz racje ziom ('t'as raison mon pote')

(7) treat: samiczka szparka zadbana?('samiczka ta chatte bien soignée?’) samiczkaa: treat pal gumy a nie.. nie dowiesz sie!! ('treat casse-toi et tu vas pas savoir')

(8) oldschoolowy: dobra zawijam ('bon je me casse')

Les exemples évoqués témoignent d'une certaine volonté des chateurs de diminuer l'écart entre l'écrit et l'oral. Les internautes s'expriment donc à travers le langage courant, et ils écrivent de la même manière qu'ils parlent entre eux, c'est-à-dire sur un ton familier et léger constituant une fonction socialisante très importante. Elle s'exprime dans le sentiment d'appartenance à ce groupe chatien. 


\subsubsection{Vulgarismes}

Le chat contenant tous types de lexiques non conventionnels, il semble aller de soi qu'il présente aussi des unités lexicales vulgaires malgré les règles d'éthique contre-indiquant leur usage sous peine d'être exclu comme dans l'exemple (10). Ainsi, en français, on rencontre des termes tels que : bordel (en tant que juron : Bordel!); salope (femme de mauvaise vie) ; à la con (grotesque, ridicule) ; s'en branler (ne pas être intéressé) ; $p d$ (pédé, 'homosexuel'). Voici quelques exemples d'énoncés :

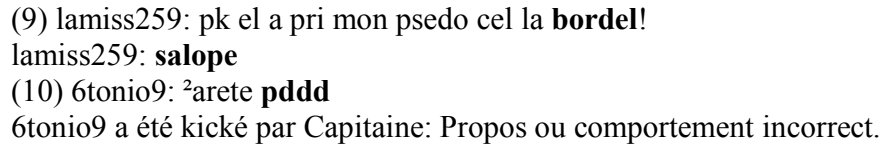

De la même manière, on rencontre des termes espagnols tels que coño (au sens propre indique 'l'organe sexuel féminin'. Expression exclamative employée pour indiquer 'la surprise' ou 'la colère'); estar de coña ('déconner') ; Hostia ('qui est considéré malsonnant par son irrévérence'; hostia veut dire 'hostie'); cabrón, cabronazo ('enfoiré, salaud') ; gilipollas ('con'); manguera (métonymie de 'bite'); me cago en tu madre ('je chie sur ta mère'). Enfin, pour ce qui est des vulgarismes polonais, on a pu noter quelques exemples comme osrać ('chier') ou opierdalać ('être fainéant') :

(11) samiczkaa:): treat nie takim tonem bo ktos cie osra kiedys makaronem ('treat pas sur ce ton parce que quelqu'un te chiera dessus avec des pâtes')

(12) Za du many ;-): a co się będę opier dalac ('et pourquoi je serais un branleur')

Par ailleurs, un phénomène particulier est observable concernant certains usages des vulgarismes. Il s'agit de l'auto-censure des chateurs. En effet, pour ne pas être exclu du chat pour propos incorrects, les chateurs ont recours à différentes stratégies visant à remplacer certaines lettres par des astérisques, des points de suspension, d'autres lettres dans les différentes langues étudiées. Les chateurs ont également recours à des troncations ou à des espacements entre les lettres ou les syllabes du mot vulgaire. Ainsi, des termes vulgaires peuvent être écrits comme suit : $p^{* * * *}$, pxte, pu ta, put4 (pute), mei9rda (merde), kur, kur wa (kurwa, 'putain').

\subsubsection{Interjections}

Comme dans les conversations orales du quotidien, les chateurs expriment une facette émotive à travers des interjections diverses. Parmi les interjections, on relève des cris et des onomatopées. Voici quelques exemples provenant du chat français :

(13) slr722t: Youhou ! Héhé, Ca va Chatter !

(14) belge80: je vous pisse dessus bande de frustré

janus6: oulala lebelge 80 je crois qu elle 1 a $\mathrm{mm}$ pas trouvée!

(15) brascute: en tout cas ce cella que je prefere c'est ma salade bukylove

brascute: pas trop de vinaigre et peu de sel hummm

(16) TAxLExSEUMxVIIICTIME : atchouuuum

(17) TAxLExSEUMxVIIICTIME: c nul

TAxLExSEUMxVIIICTIME: que des mec pfff

En espagnol, les interjections se présentent ainsi :

(18) iraultza3: wenas, libelulalibre , ja mia ('salut libelulalibre, ma fille') libelulalibre: jajaj iraultza3 ays madre ('hahaha iraultza3 aïe mère')

(19) Astronoma: porcierto mmm si, voy a estirarme en la cama ('mmm oui, je vais m'étirer sur le lit')

(20) sagara2: Lara112 te he dejao asombrada como siempre ehhhhhhhhhh jajajaja

('je t'ai laissé stupéfait comme d'habitude non hahaha')

(21) JayRex: Swang esta en la sala ('elle est dans la salle')

Swang: SI LA VEO JayRex pero estaba con ella por el privado y de repente ploff. desaparecido, otra TOC, jejeje ('oui je la vois JayRex mais j'étais avec elle en privé et d'un coup pouf. disparu, l'autre toc, hehehe') 
Et en polonais, il peut s'agir d'interjections telles que :

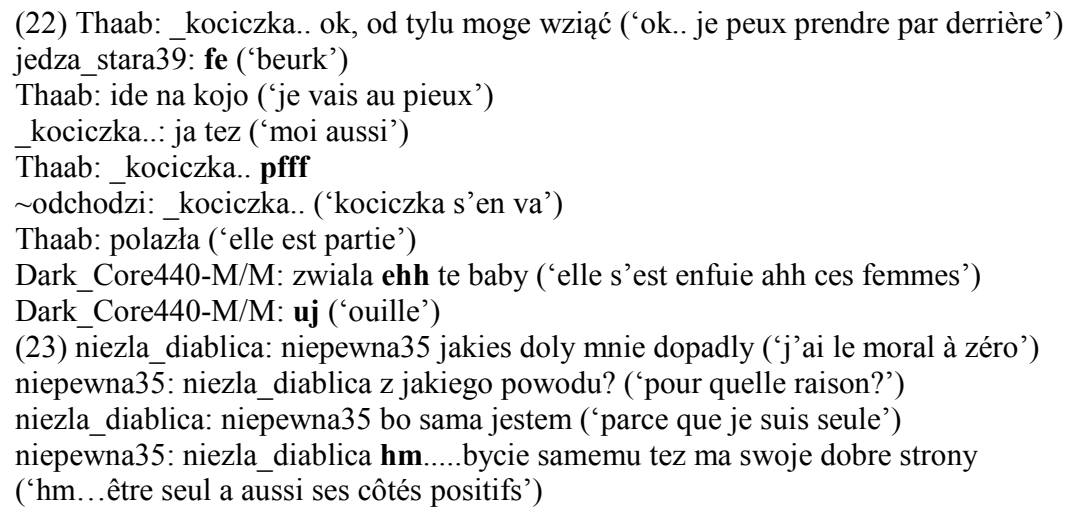

Il est intéressant de voir que les trois langues présentent des interjections similaires comme hum, mmm, $h m$, s'écrivant de façon différente mais se prononçant de manière comparable. De même, les trois langues ont l'interjection pfff en commun.

Par ailleurs, Gadet appelle une certaine catégorie d'interjections « appuis du discours » (1992: 32). Il s'agit de termes non signifiants présents dans des conversations spontanées. Plusieurs appuis du discours sont remarquables dans le chat français comme bon, ben, hein, heu, etc.

(24) Akhena15: Bon moi vais à la douche je reviens plus tard ! A toutee

(25) papillon-fleurs: vi et toi colibri0712 y a longtemps hein

(26) 93_RAISON_2PA_TCALCUL: heu mon ancien pseudo c'était MENACE 2 HAINE

En espagnol, on a pu relever les appuis du discours dans les exemples que voici :

(27) johior223: pues aqui creo solo se conoce la de carne ('eh bien ici je pense que l'on connait seulement celle à la viande')

(28) porcierto: a mi por ejemplo me alimenta mucho un beso sigiloso y suave bordeando los labios ... Astronoma ('moi par exemple ce qui me nourrit c'est un bisou discret et doux bordant les lèvres')

Astronoma: porcierto mmm eso te alimenta? yo es que sin ketchup no me alimenta na ('mmm ça te nourrit? Ben moi sans ketchup rien ne me nourrit')

(29) moilolita: oye k yo toy concentrada don_matias , este me lanza cosas, pero yo a lo mio, a sobarme y a echarte el humo y el aliento don_matias ('écoute, moi je suis concentrée don_matias, celui-là il me lance des choses, mais moi je m'en fous, je me tripote et te jette la fumée et l'hâleine don_matias') juan-carlos-I: moilolita mira que eres bruta ('t'es une brute tu vois')

Enfin, le phénomène est également remarquable dans le chat polonais :

(30) Faza(PL): to nic ide sobie ('bon rien je m'en vais')

(31) pierdolniety ;] ;]: dobra pora na kime ('bon c'est l'heure de se pieuter')

\subsection{Base argotique}

Dans le lexique à base argotique, on distingue un argot typé « jeune » de termes argotiques affectés d'un procédé formel, pour reprendre les termes de Goudaillier (2001 : 17), le verlan.

\subsubsection{Argot des jeunes}

En ce qui concerne les mots et les expressions argotiques propres aux jeunes, nous pouvons relever plusieurs termes dans le cas du français ${ }^{2}$ : boloss (victime); bogossitude (< bogoss ; beau gosse ; il s'agit 
d'une personne belle ou douée pour faire quelque chose); mater (regarder); taffer (travailler); jgalere (j'ai des difficultés); Des barres (c'est drôle); ma geul (ma gueule; mon copain); Mitos (menteur); Tailler (insulter); Clashé (insulté); Rageux (énervé, personne insultant les autres chateurs). Voici quelques exemples de ces termes en contexte :

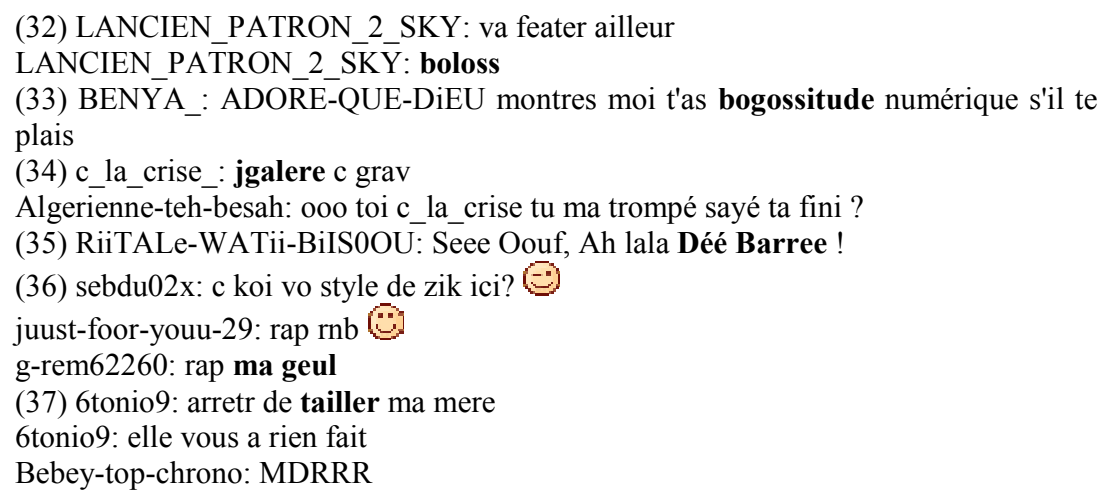

Il est à remarquer que dans le cas de bogoss provenant de beau gosse, nous assistons à une soudure entre les deux mots d'origines et qui sont graphiquement simplifiés. Dans ce cas, il s'agit d'une lexicalisation, et par là, d'une création d'un mot d'argot, d'une expression banale utilisée par les jeunes. Enfin, on ne connaît pas à ce jour la provenance du mot boloss, mais on pourrait supposer qu'il a été construit par analogie à bogoss. En effet, ces termes ont une signification s'opposant sensiblement, même s'ils ne se présentent pas comme de parfaits antonymes.

Par ailleurs, l'influence des adolescents sur les conversations des chats est très significative. Comme le constate Dejond (2006 : 32), les adolescents réinventent les mots et les expressions afin de créer leur propre identité, ce qui a toujours eu des répercussions sur la langue. Le langage des jeunes se caractérise ainsi par la fonction identitaire aussi bien que par la fonction crypto-ludique dont parle Goudaillier dans son dictionnaire du français contemporain des cités (2001).

\subsubsection{Formes argotiques verlanisées}

Les termes argotiques verlanisés présentent un critère formel clair et incontestable sur le plan de sa classification étant donné l'utilisation d'un procédé argotique connu. Ce phénomène consiste à inverser les syllabes ou bien les sons consonantiques et vocaliques dans le cas des mots monosyllabiques (Goudaillier, 1997 : 80-81) et de certains disyllabiques Il est à noter que si les termes verlanisés sont souvent utilisés par les jeunes, les mots ci-après se sont généralisés à travers des catégories de population de différents âges. Voici quelques termes relevés dans le chat français : meuf (femme); chelou (louche, bizarre, étrange) ; kéblo (bloqué) ; ouf (fou) ; renoi (noir).

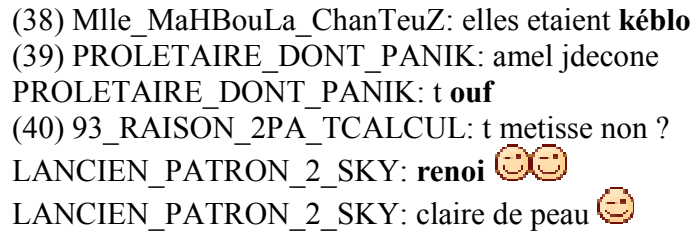

Précisons que ni le chat espagnol, ni le chat polonais ne présente de verlan qui semble être une particularité française dans le corpus étudié. 


\section{Fonds chatien}

Le fonds chatien se caractérise par la diversité des phénomènes lexicaux présents dans le chat. Ces phénomènes peuvent venir des discours oraux ou encore du lexique développé par la cybercommunauté. On énumère ainsi les phénomènes d'abréviation par troncation, de siglaison, de dérivation, et d'emprunt.

\subsection{Abréviation par troncation}

L'analyse de l'abréviation peut être distinguée en trois types de procédés : l'apocope (coupure de la fin d'un mot), l'aphérèse (coupure du début d'un mot), et la syncope (chute d'un ou plusieurs phonèmes à l'intérieur d'un mot). Ce phénomène oral s'apparente au besoin d'économie présent dans le chat. Le modèle de formation décrit joue un rôle très significatif dans notre corpus.

\subsubsection{Apocope}

L'apocope est un procédé qui fait partie du langage courant. Rappelons après Mejri (1995: 111) que l'abréviation a donné naissance à des affixes nouveaux comme " auto » < automobile ; "vélo » < vélocipède, "télé " < télévision. Le phénomène d'apocope peut être relevé dans les chats des trois langues étudiées. Deux types de coupures peuvent être observés : la coupure après une consonne et la coupure après une voyelle. C'est le cas notamment en français, où l'on trouve les deux possibilités. Ainsi, la coupure peut avoir lieu après une consonne comme par exemple : biz (< bisous); vac (< vacances); cam (< caméra).

(41) gandalfLS: bye bye babykass8669 . biz

(42) janus6: lebelge 80 tu dois pas faire pipi? ça nous ferait des vac pdt $1 / 2 \mathrm{~h}$ le tps que

tu 1 as trouve

(43) laproduxxl: cherche vrai fille chaude pour cam to cam

laproduxxl a été kické par Capitaine: va calmer tes ardeurs ailleurs !

Il est possible aussi que la coupure ait lieu après une voyelle comme dans : cata ( $<$ catastrophe); re $(<$ rebonjour); came (< camelote, 'drogue'); abdo (< abdominaux); pseudo ( $<$ pseudonyme) ; sympa (< sympathique).

Il est à noter que les exemples vac, cam et re semblent être plus spécifiques au chat que les autres exemples cités qui existent déjà depuis longtemps dans les usages. À côté de la coupure traditionnelle, il est à noter le cas du mot médoc. On peut supposer que ce terme a été coupé en méd avant d'être suffixé en $-o c$. Il s'agit donc d'une resuffixation après apocope tout comme les termes modo venant de «modérateur ", travello venant de «travesti » en français, et privi venant de "privado » en espagnol sont resuffixés après apocope.

En somme, il faut signaler que le procédé de raccourci est utilisé depuis longtemps, mais il semble prendre de l'ampleur dans certains groupes sociaux. Ainsi, sur le chat, tronquer, abréger et raccourcir a pour objectif d'économiser l'espace graphique occupé dans le discours. On peut également supposer que l'oral influe dans ce cas sur l'écrit.

En espagnol on note la prédominance du cas de la coupure après une voyelle comme dans les exemples suivants : pa (< para ; 'pour') ; na (< nada ; 'rien') ; se pue (< se puede ; 'se peut') ; funci $(<$ funcionarios ; 'fonctionnaires'). Notons aussi le cas du mot finde ( $<$ fin de semana; ; 'weekend') où l'on observe la chute du lexème semana et l'agglutination des segments fin et de dans le syntagme figé fin de semana. D'autre part, la coupure après une consonne semble plus rare. On a seulement pu relever super ( $<$ supermercado ; 'supermarché').

Le polonais présente aussi une certaine tendance à abréger les mots, ceci en exploitant les deux procédés mentionnés. Ainsi, la coupure peut se situer après une consonne : kam (< kamera ; 'caméra'); godz (< godzina; 'heure') ; Aleks ( $<$ Aleksandrów ; le nom de la ville polonaise) ; komp $(<$ komputer ; 'ordinateur'). La coupure peut aussi se situer après une voyelle : cze (< cześć ; 'salut') ; si $(<$ się ; particule 
« se ») et alko (< alkohol ; 'alcool') ; dowi (< dowidzenia ; 'au revoir') ; ta (< tak ; 'oui') ; spoko (< spokojne ; 'tranquille').

De manière générale, précisons que comparativement aux chats français et polonais, les apocopes après une consonne semblent être moins fréquentes et productives en espagnol.

\subsubsection{Aphérèse}

L'aphérèse est remarquable dans les trois langues analysées et se manifeste selon les deux types de coupures déjà observées dans le cas des apocopes, c'est-à-dire après consonne ou après voyelle. Le phénomène est donc comparable, bien que moins présent. Selon Goudaillier (2001: 27), le phénomène d'aphérèse "prend (...) de plus en plus d'importance par rapport à l'apocope ", notamment en ce qui concerne les usages argotiques des jeunes. Examinons les exemples d'aphérèses relevés dans nos trois corpus. En français, on note des coupures après une consonne comme dans touze (< partouze, séance collective de débauche sexuelle) ou net (< internet).

(44) lorenzo-11: $\mathrm{d}$ femme pr cam chaud envi d une touze

(45) KillyEkFael: naamah j'ai rien contre le net, tout 1 contraire, c'est les tchats que je vais arréter

On relève aussi des coupures après une voyelle : bleme $(<$ problème) ; jour ( $<$ bonjour) ; lut $(<$ salut $)$.

(46) flavien76: re des bleme

(47) ninifer: slt tous le monde

herve92: ninifer lut

Le phénomène est similaire en espagnol. Il existe des coupures après une consonne : tas (< estás ; 'tu es') ; ta luek (< hasta luego ; 'à bientôt') ; net (< internet).

(48) kelben: que tal tas hoy pasabaporaquiyviluz ? ('comment vas-tu pasabaporaquiyviluz ?')

(49) topacio2020: falla el telefono y la net ('le téléphone et le net coupent')

On observe des coupures après une voyelle : jamía (< hija mía ; 'ma fille’) ; jomío (< hijo mío ; 'mon fils').

(50) navi_2277: mandamela jomio mandamela igle67 ('envoie-le moi mon fils envoie-le möi igle67')

Le polonais présente les mêmes types de coupure. La coupure après une consonne comme dans net $(<$ internet), et la coupure après voyelle comme dans bry wieczór (< dobry wieczór ; 'bonsoir').

(51) Adrian18Lodz..,,: angie utni ('angie coupe')

angie: net? ('le net?')

(52) SALWIA18: bry wieczor

Nous observons un cas particulier dans les trois langues analysées, à savoir la forme abrégée d' "Internet », net. En effet, inter est un préfixe, et contrairement aux autres cas, il s'agit d'une unité significative, et non pas d'une syllabe. Il s'agit donc d'un mot diffusé internationalement. Précisons aussi qu'un cas espagnol présente à la fois apocope et aphérèse dans la même séquence graphique, à savoir ta luek ( $<$ hasta luego).

\subsubsection{Syncope}

Le phénomène de syncope n'a pu être relevé que de façon ponctuelle dans les chats français, espagnol et polonais. En français, on note $m c i$ (merci). Notons que seules trois occurrences du mot mci apparaissent dans le chat. De même, en espagnol on relève tos (< todos; 'tous'). En polonais, on observe nastki (< nastolatki ; 'adolescentes') et czloku (< człowieku; sens propre 'homme', vocatif). Dans l'exemple 
polonais $c z ł o k u$ (< człowieku), on remarque que la syncope consiste en l'amuïssement d'un phonème (wie dans człowieku ) ou de deux (to-lat dans nastolatki) à l'intérieur d'un mot.

En définitive, les particularités lexicales citées consistent à supprimer des parties de mots sans en altérer la compréhension. Les chateurs ont recours à la troncation, phénomène populaire ancien qui influence le standard. Ce phénomène connu et récursif permet l'économie de temps et d'espace. Parmi les procédés d'abrègement cités, c'est l'apocope qui reste la plus utilisée par les internautes chateurs. Ce procédé de troncation est d'ailleurs celui qui est le plus répandu dans l'argot traditionnel.

\subsection{Siglaison}

Dans le cas des chats, les sigles présentent une utilisation élargie car ils transmettent un sens et une émotion, et ne font pas que renvoyer au nom d'une structure. Deux catégories de sigles propres au chat ont pu être observées dans notre corpus : les sigles à épeler et les acronymes.

\subsubsection{Sigles à épeler}

Dans le chat français, les sigles à épeler sont ceux qui sont les plus diversifiés. On peut relever les sigles suivants : $m d r$ (mort de rire), $p t d r$ (pété de rire), xpdr (explosé de rire), ainsi que les variantes PTDxxx, $m d r r$ ' et $m d$ 'rrr. Voici quelques exemples :

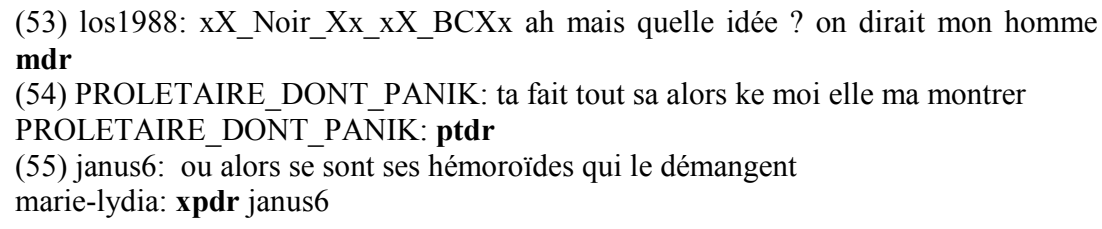

Il est à noter que $p t d r$ est rarement prononcé de façon totalement épelée par les usagers, mais que les mots représentés par les deux dernières lettres, c'est-à-dire «de rire » sont généralement prononcés dans leur intégralité. D'autre part, $x p d r$ est un sigle épelé intégralement avec une prononciation à l'anglaise concernant le « $\mathrm{x}$ ». Dans ce sigle, $x p$ est un squelette consonantique de « explosé », et $d r$ signifie " de rire » comme dans le cas de $p t d r$. Précisons que le polonais présente différents cas de sigles à épeler empruntés à l'anglais tels que $d n b$ (drum and bass) et $b t w$ (by the way).

\subsubsection{Acronyme : cas de lol}

Dans les trois chats analysés, apparaît un sigle acrophonique $L O L$ provenant de l'expression anglaise laughing out loud qui signifie littéralement rire à voix haute. Il est possible d'observer que ce sigle peut être confondu avec un lexème. En effet beaucoup de jeunes ne connaissent pas la signification exacte du sigle anglais, mais plutôt la signification traduite approximativement du type «rigoler», "rire aux éclats », etc. Le sigle $L O L$ est un sigle particulier. Il peut apparaître en position de verbe, comme dans l'exemple français suivant :

(56) Niko95: je lol ce chat ('ce chat me fait rire')

Il peut également apparaître en position d'adjectif :

(57) Ju922: t'es vraiment trop lol (t'es vraiment drôle)

En tant que substantif comme dans les exemples espagnols suivants où lol mio est utilisé à la place de Dios mio dans le premier cas :

(58) Helmantiquito: fly_69 falso yoooooo ?........ lol mío! Calla pecadora! ('Moi faux?

Mon dieu! Ferme-la pécheresse')

pirada01: jajaja hjfuh k brutitaaa eres un lol ('hahaha quelle brute, t'es un marrant') 
Dans ces cas, lol ne subit pas seulement un changement de catégorie grammaticale, mais un changement de statut. Il passe de sigle à lexème, et en tant que lexème, il conserve une forme unique dans les différentes positions grammaticales. $L O L$ fait donc preuve du dynamisme du langage. De plus, les supports modernes tels que les chats, les blogs, et les SMS grâce auquel ce terme est apparu, ont provoqué le passage de l'écrit à l'oral ${ }^{3}$ chez certains jeunes Français qui l'utilisent beaucoup dans leurs pratiques langagières écrites. Notons enfin que nous avons pu repérer quelques variantes graphiques de $L O L$ dans le chat français, à savoir loul, lool et loool, introduisant une dimension ludique s'ajoutant au sens initial du sigle.

Par ailleurs, le sigle $L O L$ est évidemment utilisé en tant que sigle, ce qui représente d'ailleurs la quasiintégralité des usages puisque seuls les quelques exemples cités précédemment dans le cas du français et de l'espagnol présentent la particularité de lexicaliser l'acrophone. Des exemples provenant du corpus des différents chats peuvent être cités. En français d'abord, $L O L$ peut être utilisé pour s'exprimer par rapport à ce qui a été dit précédemment dans la conversation et/ou pour remplacer l'onomatopée haha :

(59) EKyA: si je chante tout le monde se barre

lady-gaga-Offficial: lol

(60) Bebey-top-chrono: 5 heures après, tu réponds lol

On relève le même type d'exemples dans le corpus espagnol :

(61) Princessasil: tu no sabes apreciar la belleza interior lol ('tu ne sais pas apprécier la beauté intérieur lol')

Le polonais présente le même usage :

(62) Wariateczka : lol alle dziecinada ('lol que d'enfantillages')

Le sigle $L O L$ est le seul sigle universel, c'est-à-dire qu'il est récurrent quelle que soit la langue pratiquée dans l'espace chatien.

\subsection{Dérivation}

Fréquemment utilisé dans les chats, le phénomène de dérivation se présente sous la forme d'affixation qui consiste en l'adjonction au radical de suites phoniques antéposées ou postposées.

\subsubsection{Préfixation}

Dans le chat, le phénomène de préfixation est très ponctuel. Le français et l'espagnol présentent le même cas de préfixation en re-. Dans les deux cas, cela signifie littéralement «à nouveau », mais lorsque re- est utilisé seul dans le discours, cela signifie en français « rebonjour », «je suis de retour » et en espagnol, « rebuenas » orthographié rebuenasss dans le corpus de chat. En revanche, le polonais ne présente pas ce phénomène.

\subsubsection{Suffixation}

Les suffixes, utilisés plus régulièrement, renvoient souvent à des catégories générales comme les suffixés diminutifs et augmentatifs à tonalité méliorative ou péjorative. Ces morphèmes lexicaux sont utilisés pour transmettre des informations relatives aux émotions éprouvées par les chateurs.

Bien que la langue française offre une certaine variété des suffixés en question, sur le chat, on n'en observe que quelques-uns, dont un type de diminutif mélioratif français, -ette dans memelouиииииииипеeеeеette.

On peut aussi relever en espagnol des exemples comportant les suffixes : -ito, -ita, -illo qui introduisent une tonalité hypocoristique méliorative : calorcito ( $<$ calor ; 'chaleur' + ito) ; fresquito ( $<$ fresco ; 'frais' + ito) ; brutita (< bruta ; 'brute' + ita) ; lastimita (< lástima ; 'peine' + ita) ; chiquillo (< chico ; 'garçon’ + illo). 
D'autre part, en polonais, on relève de nombreux suffixes mélioratifs comme : -ik, -utki, -ek, -iczka, -atko, -usek, -ecia, -ka: karpik (< karp ; 'carpe' + ik, ici 'une fille sexy'); fajniutkie (< fajny ; 'cool' + utkie) ; dzionek (< dzień ; 'jour' + ek) ; samiczka (< samica ; 'femelle' + iczka) ; zdjatko (< zdjęcie ; 'photo' + łątko) ; lizusek (< lizus ; 'flagorneur' + usek) ; brunecia (< brunetka ; 'brune' + ecia) ; dobranocka (< dobranoc ; 'bonne nuit' + ka). La diversité des suffixes polonais est très importante et comme dans le cas de l'espagnol, elle introduit un effet familiarisant renforçant la proximité entre les chateurs. Enfin, la diversité des suffixes des trois langues étudiées provient chaque fois des discours oraux.

Par ailleurs, on observe l'utilisation de suffixes augmentatifs. En espagnol, on peut citer des augmentatifs mélioratifs tels que : besazo (< beso ; 'bisou' + azo) ; guapetona (< guapa ; 'jolie' + etona) ; besote ( $<$ beso ; 'bisou' + ote) ; guapísimo (< guapo; 'joli' + isísimo). De même, en polonais, on relève l'exemple narazicho (< narazie ; 'à bientôt') dans l'exemple oki narazicho wszystkim ('ok à bientôt tout le monde'). En espagnol comme en polonais, les suffixes augmentatifs visent à amplifier le sens des mots utilisés. Ils expriment donc une émotion particulière. D’autre part, aucun exemple de suffixes augmentatifs n'a pu être relevé dans notre corpus français.

Par ailleurs, on relève différents suffixes augmentatifs péjoratifs. Tout d'abord, en espagnol on observe cabezota (< cabeza ; 'une grosse tête' + ota) et gentuza (< gente ; 'gens' veut dire 'racaille' + suffixe uza). Ensuite, le chat polonais présente les augmentatifs péjoratifs suivants : dupsko ( $<$ dupa ; 'cul' + resuffixation -sko après la chute de la voyelle « a ») ; cielsko (< ciało ; 'corps') ; ciacho (< ciastko ; 'gâteau' + acho, ici 'un beau gosse').

Notons qu'un cas à part existant déjà indépendamment du chat a pu être relevé en français car il s'agit d'un suffixe péjoratif qui n'est ni diminutif, ni augmentatif : salopard ( $<$ salop + suffixe - ard).

Après avoir réalisé notre analyse descriptive, il convient de souligner que l'emploi de suffixes modificateurs est bien présent dans les conversations des internautes. Les morphèmes de dérivation en question changent plus ou moins sensiblement le sens d'origine des lexèmes concernés. Le rapport entre les chateurs étant souvent détendu, et l'écran de l'ordinateur introduisant une certaine distance, les chateurs utilisent beaucoup de procédés de constructions afin de pallier l'absence physique d'une conversation orale de la ou des personne(s) à laquelle (auxquelles) ils parlent. Ces suffixes ont donc un rôle familiarisant face au côté abrupt et froid dégagé par le support technique, permettant une meilleure intégration dans la communauté chatienne. D'ailleurs, les chats étant des espaces de détente pour les chateurs, ces stratégies de constructions lexicales permettent d'introduire une tonalité agréable dans les discours. Par opposition, les suffixes péjoratifs, peu nombreux, ont une valeur sémantique plus marquée, lorsqu'ils apparaissent.

\subsection{Néologie par l'emprunt}

Dans le chat, on constate des emprunts majoritairement à l'anglais, mais aussi à d'autres langues.

\subsubsection{Anglicismes}

Les emprunts à l'anglais semblent posséder un pouvoir de modernité, de secret, de sentiment de maîtriser un langage particulier et d'appartenir à un groupe. Dejond souligne le fait que dans le cyberlangage, ils traduisent un signe d'appartenance à une communauté qui a envie de se comprendre, aussi paradoxal que cela puisse paraître a priori (Dejond, $2006: 45$ ).

Le langage cyber contient des anglicismes sous formes de mots, mais aussi des phrases entières qui sont parfois écrites en anglais, ce qui constituerait une preuve de l'importance et de la vivacité de cette langue dans le chat. Il est utilisé dans tous les types de relations modernes. Voici quelques mots que l'on peut observer régulièrement dans les chats des trois langues étudiées : hello ('salut') ; bye ('salut'); sorry ('désolé') ; shit ('merde') ; hot ('chaud'); ok ('d'accord') ; cool ('génial'); kiss ('bisous') ; big fish ('grand poisson') ; miss ('mademoiselle') ; top model ; mister gay ('monsieur mec') ; new ('nouveau') ; top secret. 
Par ailleurs, parmi les phénomènes d'anglicisation remarquables dans les conversations chatiennes, il convient de souligner que "l'emprunt ou l'adaptation de la terminologie technique anglophone, (...) $n$ 'a rien d'étonnant, dans la mesure où les environnements logiciels (...) restent en langue anglaise » (Anis, 2000 : 67). En d'autres termes, il s'agit des mots d'Internet utilisés et diffusés dans les chats pour des besoins techniques et communicatifs. À titre d'exemples, les termes ci-après évoquent les référents propres à ce milieu : $P C$ (personal computer); beug (vient du mot anglais bug); flood (flooder: le fait d'envoyer une telle quantité de messages dans un canal ou une boîte à lettres, que celui ou celle-ci sera saturé(e) et deviendra inutilisable); room ('chambre'); fake ('un faux; personne se présentant sous une fausse identité sur Internet'); nolife ('personne dépendante des jeux en réseau et d'Internet'). Les exemples cités démontrent la fonction cryptique des anglicismes employés ; ils sont en effet incompréhensibles pour ceux qui n'appartiennent pas au « cybergroupe ».

Les internautes insèrent également des phrases entières telles que :

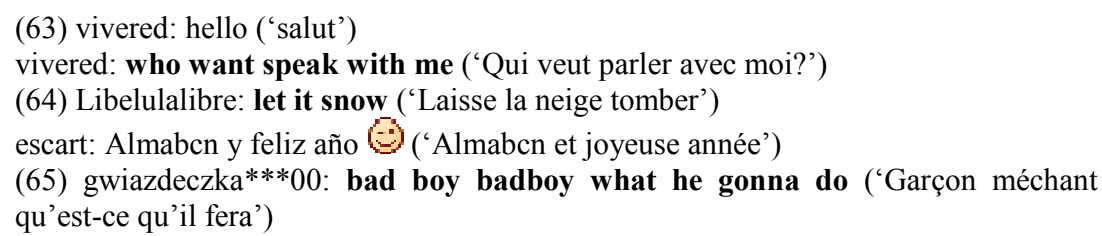

Nous remarquons que ces phrases sont toutes écrites intégralement en anglais dans des chats qui ne sont pas des chats anglais. Il est donc à supposer que la langue anglaise a à la fois pour rôle d'introduire un effet de différenciation visant à interpeller la communauté des chateurs présents dans le chat en question, et de renforcer la proximité entre les internautes chateurs par un aspect identitaire relatif à l'emploi de la langue anglaise.

Dans le cas du polonais, certains mots anglais sont transcrits à la manière polonaise. Il s'agirait en quelque sorte d'emprunts hybrides anglo-polonais, où la base lexicale est anglaise et où la graphie est polonaise. Dans ce cas, la graphie polonaise est simplifiée comme dans szit pour shit ou encore comme dans les exemples suivants : bay (< bye ; 'salut') ; orajt (< all right ; 'd'accord') ; helot (< hello ; 'salut') ; kaman (< come on ; 'allez'); siarap ( $<$ shut up ; 'tais- toi') ; fenks ( $<$ thanks ; 'merci') ; not koment $(<$ no coment ; 'pas de commentaire') ; lovelka, lovka ('amoureux') ; Endriu (< Andrew ; 'André, Adrien' ) ; cya (< see you ; 'à bientôt') ; plis (< please ; 'je t'en prie').

Par ailleurs, les anglicismes apparaissent également au niveau syntaxique comme dans :

(66) veilside-hks: tu cliques sur mon pseudo et il y a aller voir le profil c'est no problem pour le voir ('il n'y a pas de problème pour le voir')

(67) brunet cjetnyyyy: CHEERLADEKA ZADZWONIE DO CIENIE ('Cheerladeka je vais t'appeller')

(68) brunet_cjetnyyyy: WCZORAJ BYLAS OUT OF ORDER ('Hier tu as été absente')

Ces énoncés semblent suggérer des formes créatives de la langue produites spontanément à l'écrit. Ainsi le langage du chat présente les traits d'un pidgin, même si ce type de langage ne s'est pas formé grâce au contact des groupes parlant différentes langues.

\subsubsection{Emprunts aux autres langues}

À part les anglicismes, plus rares sont dans le langage cyber les mots provenant d'autres langues. Leurs emplois semblent être plutôt liés au sujet de la discussion. Ainsi dans le chat à propos de la prononciation espagnole, on relève des mots et des locutions espagnoles, ce qui serait probablement une sorte d'amusement. Par exemple,

(69) Sandrine: Salut bonnitaa ('jolie’)

Sandrine: Quand je suis allée en Espagne, je n’ai jamais pû commander le « zumo de naranja »!! ('jus d'orange') 
(70) Sandrine : Je rêverais d'avoir l'accent espagnol

Sandrine : C'ets vraiment très joli

Fran_vlc : lol pas pour moi, et je suis espagnol

Richard : Si! Tienes razón ! :P ('Oui, t’as raison')

Il est à préciser que les quatre dernières répliques citées représentent un fragment de conversation à propos de l'accent espagnol. Les mots espagnols introduits par le chateur Richard résultent donc de la thématique de la conversation en cours. Ceci étant, le corpus français présente parfois des emprunts à l'arabe comme kiffer signifiant «aimer, adorer» ou encore salam alikoum, alikoum salam, ou aleikouuum seleeem signifiant « bonjour, salut ».

Dans le corpus espagnol, on note une présence des langues co-officielles d'Espagne telles que le catalan (par exemple : no pasa res ('c'est rien'); ningú em parla? ('personne me parle')) et le basque (par exemple : agurrrr ('au revoir')).

(71) riesgo29: Kuiper gracias pero si no pasa res lo puse de broma ('Kuiper merci mais c'est rien je l'ai mis pour rigoler')

(72) teclas63: iraultza3 lliurem otroporfavor2 fly_69 Alma2 que me voy agurrrrr

('iraultza3 lliurem otroporfavor2 fly_69 Alma2 'je m'en vais au revoir')

Il est également possible de noter l'emploi de structures de l'espagnol d'Amérique Latine comme de donde sos (de dónde eres; 'tu viens d'où') ; vos (tú ;'toi'). On relève aussi ponctuellement des emprunts à l'allemand comme wie gets (wie geht's ; 'comment vas-tu') et au Portugais comme obrigada ('merci') avec se variantes : moito obrigada (muito obrigada; un grand merci), obrigadissima (le mot portugais obrigada + suffixe intensificateur -íssima existant aussi en espagnol ; 'un grand merci').

Remarquons l'étirement graphique de agurrrr et la simplification graphique de la séquence allemande geht's par gets. Pour ce qui est des mots étrangers dans les conversations en polonais, les exemples suivants peuvent être indiqués : todos (salut tout le monde); ahoj ('salut' en tchèque) ; merci.

Il est intéressant de souligner la présence du mot italien « ciao » dans les trois langues analysées. C'est le chat espagnol qui offre la plus grande variété graphique du mot en question: chaooo, chauuu, chaucitooo, сіаииии. Les salons de bavardage en France et en Pologne se limitent à la graphie italienne : ciao !

\section{5 Émoticônes (ou smileys)}

Les émoticônes complètent les mots du discours dans la mesure où elles expriment des émotions et où elles apportent un sens fort. Deux types de structures d'émoticônes peuvent être distingués : les émoticônes textuelles et les émoticônes-images ${ }^{4}$.

\section{1 Émoticônes textuelles}

Les émoticônes textuelles sont des petits visages composés à l'aide des signes du clavier de l'ordinateur. La base est simple : les deux points «: : pour représenter les yeux, le tiret « - » pour le nez, la parenthèse fermante «) » pour la bouche en sourire, et la parenthèse ouvrante " ( " pour la bouche triste. À partir de cette base, les internautes puisent dans chaque signe qu'offre le clavier pour sans cesse réinventer de nouveaux visages, que l'on devrait lire à l'horizontale et à 90 degrés. De surcroît, il est intéressant de noter l'existence de nombreuses variantes des émoticônes de base. Ainsi, le nez peut être représenté par le signe graphique «o », comme dans :o), ou omis, comme dans :). Les parenthèses fermantes «) ) et ouvrantes «(« peuvent se répéter, intensifiant la représentation du sourire ou des pleurs des chateurs en fonction des cas. À titre d'exemple, voici quelques émoticônes textuelles : :-) :) ou =) (visage joyeux), :-( $:(=($ (visage triste), $x d X D$ ou $=D$ (rire), :'( ou ='( (pleurs), ;-) ou ;) (clin d'œil), :-* (bisous), : $p$ ou =p (visage tirant la langue). Par ailleurs, il est important de noter que certaines émoticônes textuelles sont transcrites horizontalement. C'est le cas des Japonais qui utilisent des émoticônes spécifiques qui représentent une grande variété « d'yeux » : ${ }_{-} \wedge$ pour le sourire $; T_{-} T$ pour la tristesse ; ô_Ô pour la surprise. 


\section{2 Émoticônes-images}

Les émoticônes qui au début étaient uniquement formées de signes de ponctuation ont été vite enrichies des combinaisons de chiffres et de lettres pour ensuite être de plus en plus remplacées par une image plus moderne : celle-ci est sonore, statique ou animée, et elle imite le visage, représente un personnage fictif ou résume une situation en une seule image. Ainsi, les chateurs peuvent se souhaiter joyeux anniversaire en ayant recours à l'une des images proposées dans des galeries d'émoticônes :

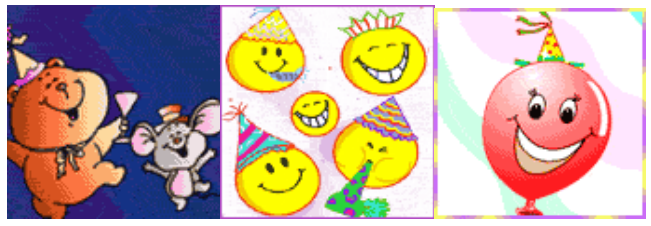

Figure 1. Images festives

Ils peuvent aussi exprimer leur amour par l'une des images proposées dans le répertoire d'émoticônes :

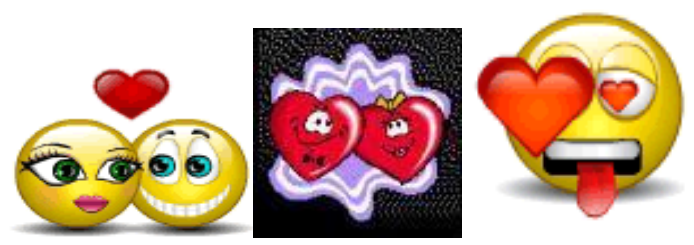

Figures 2. Images d'amour

Il convient de souligner que les émoticônes modernes représentées sous la forme d'images se lisent facilement et il semble qu'ils n'exigent pas de connaissances préalables d'un code quelconque. Parmi les émoticônes sous forme de dessins, il est également possible de relever un certain nombre de variantes

comme : 9 (le sourire et la joie), 9 (le rire), (la tristesse), $\Rightarrow$ (l'indifférence), 8 (l'air rêveur), $\equiv$ (le cri).

En définitive, les émoticônes représentent des spécificités et renforce le côté à la fois informel et relâché de ce type de communication.

\section{Conclusion}

Le chat présente un lexique particulier constitué à la fois par un vocabulaire standard, mais aussi argotique. Il n'existe pas véritablement un registre langagier propre au chat séparé de la langue « générale », mais un ensemble lexical concentré dans ces espaces cyber de discussion engendré par la diversité des chateurs. Le chat a donc la particularité de présenter le langage standard et courant des discussions spontanées du quotidien où s'enchevêtrent des mots relevant des registres argotique, familier, populaire ou vulgaire, ainsi que des mots d'Internet et quelques spécificités chatiennes. Ces lieux de communication sont le refuge d'une large diversité lexicale influencée par la transcription des langages oraux, étant donné l'accès possible et libre pour tous les internautes aux salons de discussion. Cette concentration de diversité de lexique habituellement utilisé à l'oral contribue d'ailleurs à construire l'originalité des chats car il s'agit d'un des traits majeurs, bien que moins saillant que les caractéristiques graphiques, permettant aux pratiques chatiennes de se démarquer de tout autre écrit. D'autre part, il est important de souligner que les choix lexicaux des chateurs semblent être dictés par l'émotivité et la volonté de pallier le déficit propre à l'écrit de la richesse expressive des gestes lors d'une discussion orale. Ainsi, le vocabulaire chatien se caractérise par une grande volonté de transmettre les émotions, aussi bien positive que négatives à travers l'emploi de registres habituellement oraux, et donc dynamiques, ou encore l'utilisation de procédés formels de raccourcissement transcrivant, là encore, le caractère dynamique et relâché par l'impression de 
vitesse introduite dans le discours. En effet, l'analyse lexicale démontre que les chateurs utilisent de préférence les mots formés grâce à diverses stratégies linguistiques telles que la troncation, la dérivation, la composition, la siglaison, les emprunts, ou les registres. Il est à noter que si le chat présente la particularité de rassembler dans un même lieu tous ces lexiques habituellement oraux, ce qui est un phénomène qui lui est propre, les seules véritables spécificités chatiennes, sont l'usage de jargon d'Internet pour des besoins communicatifs et techniques compréhensible principalement pour la communauté d'internautes initiés, ainsi que les sigles. En outre, on peut avancer que les sigles, et notamment lol qui est diffusé dans les chats des trois langues étudiées, ont à la fois une valeur emblématique et identitaire du chat. De la même manière, les émoticônes utilisées dans un cadre publicitaire ou informatif atteste leur caractère symbolique de ce type d'écrits. En somme, les pratiques langagières des chateurs et l'appartenance à une communauté virtuelle leur permettent de renforcer leur identité langagière. Ceci étant, nous ne sommes pas face à une communauté hermétique. Non seulement, personne n'est exclu, mais de plus la communauté virtuelle ne présente aucune frontière avec le monde réel.

En définitive, le français, l'espagnol et le polonais fonctionnent de manière similaire sur le plan des usages dans les chats étudiés. Seuls quelques phénomènes comme par exemple l'argot des jeunes, le verlan ou les affixations fonctionnent légèrement différemment d'une langue à l'autre. On constate donc que les habitudes du langage du chat sont universelles. Il ne s'agit pas d'un langage universel en lui-même, mais de pratiques langagières spécifiques aux chateurs. Ces derniers ayant des habitudes langagières dans leurs parlers, rapprochent spontanément l'écrit de l'oral. Les spécificités orales représentées par de nombreux termes de registres non standards plus ou moins anciens, s'ajoutent aux particularités chatiennes telles que les phénomènes graphiques ou les usages des émoticônes. Par conséquent, les caractéristiques orales et les caractéristiques écrites s'enchevêtrent, créant ainsi une sorte de langage interstitiel qui pourrait représenter un genre communicationnel nouveau.

\section{Références bibliographiques}

Anis, J. (1999). Chats et usages graphiques. In Anis, J. (éd.), Internet communication et langue française. Paris : Hermès. 71-90.

Anis J. (2000). Dispositifs multimédias et conversation électronique : approche sémiolinguistique. In Lancien T. (éd.) Cahiers du français contemporain. $\mathrm{n}^{\circ}$ 6. (multimédia : les mutations du texte). Fontenay St-Cloud : ENS. 57-86.

Anis, J. (2003). Communication électronique scripturale et formes langagières : chat et SMS. In Actes des Quatrièmes Rencontres Réseaux Humains / Réseaux Technologiques. Université de Poitiers. http://edel.univpoitiers.fr/rhrt/.

Anis, J. (2004). Les abréviations dans la communication électronique (en français et en anglais). In Andrieux-Reix, N., Branca-Rosoff, S., et Puech, C. (éd.), Écritures abrégées (notes, notules, messages, codes...) : L'abréviation entre pratiques spontanées, codifications, modernité et histoire. Paris/Gap : Ophrys. 97-112.

Dejond, A. (2006). Cyberlangage. Bruxelles : Racine.

Gadet, F. (1992). Le français populaire. Paris : Presses Universitaires de France. «Que sais-je ? ».

Goudaillier, J.-P. (1997).: Quelques procédés de formation lexicale de la langue des banlieues (verlan monosyllabique, aphérèse, resuffixation). In Actes du colloque «Touche pas à ma langue !(?) les langues des banlieues. Marseille : Cahier de la recherche et du développement. 75-86.

Goudaillier, J.-P. (2001). Comment tu tchatches! Dictionnaire du français contemporain des cités. $3^{\mathrm{e}}$ édition. Paris : Maisonneuve et Larose.

Goudaillier, J.-P. (2011). De l'intérêt de décrire en linguistique les parlures argotiques traditionnelles et contemporaines. In Festschrift für Thomas Stehl. Université de Potsdam. 119-128.

Lorenz, P. (2010). Le chat en tant que phénomène langagier : étude comparative français-espagnol-polonais, Thèse de doctorat, Paris/ Łódź : Université de Paris V Sorbonne.

Mejri, S. (1995). La néologie lexicale. Publications de la Faculté des Lettres de la Manouba. 
Michot, N. (2007). Les usages lexicaux des jeunes sur les supports modernes de communication, Actes du 26e Colloque International sur le Lexique et la Grammaire (session thématique), In Camugli C., Constant M., Dister A. (éd.), Université Paris-Est / Marne-la-Vallée, http://infolingu.univ-mlv.fr/Colloques/Bonifacio/proceedings/michot.pdf.

Michot, N. (2008). Les représentations graphiques du lexique dans les pratiques atypiques écrites des jeunes, Actes du CMLF 2008, le Congrès Mondial de Linguistique Française, In Durand J., Habert B., Laks B. (éd.), Paris, La Cité Internationale Universitaire : EDP Sciences. 1163-1177,

http://www.linguistiquefrancaise.org/index.php?option=article\&access=standard\&Itemid=129\&url=/articles $/ \mathrm{cmlf}$ /pdf/2008/01/cmlf08082.pdf

Yus, F. (2001). Ciberpragmática. El uso del lenguaje en Internet. Barcelona : Ariel Lingüística.

\footnotetext{
${ }^{1}$ Anis, 1999, 2003, 2004.

${ }^{2} \mathrm{Il}$ est à préciser que l'espagnol et le polonais ne présentent pas de phénomènes argotiques aussi marqués que le français au niveau de l'utilisation de lexiques propres aux jeunes dans notre corpus de chats.

${ }^{3}$ Il est à noter que les emplois oraux présentent généralement une fonction ludique.

${ }^{4}$ Cette terminologie a été élaborée à la fois à partir de ce que Yus (2001 : 128) appelle «textos icónicos » ('textes iconiques'), et des usages des internautes répandus sur la Toile.
} 